\title{
A QUALIDADE CONJUGAL COMO PREDITORA DOS ESTILOS EDUCATIVOS PARENTAIS: O PERFIL DISCRIMINANTE DE CASAIS COM FILHOS ADOLESCENTES
}

\author{
Clarisse Mosmann ${ }^{1}$ \\ Adriana Wagner ${ }^{2}$ \\ Jorge Sarriera ${ }^{3}$
}

Resumo: O presente estudo buscou traçar um perfil discriminante de casais entre as dimensões da qualidade conjugal, adaptabilidade, coesão, satisfação, nível de conflito conjugal, as variáveis sócio-demográficas, e os estilos educativos parentais exercidos com os seus filhos. Para tanto utilizou-se uma amostra de 149 casais com, no mínimo, um filho adolescente, de nível sócio-econômico-médio, residentes na cidade de Porto Alegre e no interior do Rio Grande do Sul. Foi utilizado um instrumento composto de quatro escalas: três para mensurar as dimensões da conjugalidade e uma para as dimensões da parentalidade. Os resultados mostraram que as dimensões da qualidade conjugal se expressam de forma efetiva e dinâmica nas variáveis que compõe os estilos educativos parentais. Ademais esses resultados mostraram uma coerência de funcionamento entre as características individuais dos cônjuges, tanto no exercício da conjugalidade, quanto da parentalidade.

Palavras-Chave: família, qualidade conjugal, estilos educativos parentais, relações entre subsistemas.

Marital quality as a predictor of parental rearing styles: the discriminant profile of couples with teenager children (Abstract): The present study aimed to draw a discriminating profile of couples taking into consideration the dimensions of marital quality, adaptability, cohesion, satisfaction, level of marital conflict, the social-demographic variables and the parental rearing styles practiced with their children. We used a sample of 149 middle class couples with at least one teenage child, living in Porto Alegre city or in the countryside of Rio Grande do Sul. It

\footnotetext{
${ }^{1}$ Doutora em Psicologia pela PUCRS/Brasil. Terapeuta de Casal e Família. Email: clarisse@redemeta.com.br

2 Doutora em Psicologia pela UAM/Espanha. Professora da PUCRS/Brasil. Coordenadora do Grupo de Pesquisa "Dinâmica das Relações Familiares" PUCRS. Terapeuta de Casal e Família.

${ }^{3}$ Doutor em Psicologia pela UAM/Espanha. Professor da UFGRS/Brasil.
} 
was used an instrument composed of four scales: three to measure the dimensions of marital relationship and one for the dimensions of parenting. The results showed that the dimensions of marital quality express themselves in a dynamic and effective way in the variables which compose the parental rearing styles. Moreover, these results showed a coherence of functioning between the individual characteristics of the spouses concerning the practice of marital relationship as well as parenting.

Keywords: family, marital quality, parental rearing styles, subsystem relations.

\section{Introdução}

Atualmente um grande número de estudos tem documentado as associações entre a qualidade do relacionamento conjugal e a relação parental. Especificamente, sabe-se que algumas dimensões da conjugalidade, como o conflito conjugal, pode expressar-se em práticas educativas coercitivas e com pouca proximidade afetiva. Entretanto, essa conexão acaba por não explicar a natureza dessas associações (Frosch \& Mangeldorf, 2001); (Buehler \& Gerard, 2002); (Davies, Sturge-Apple \& Cummings, 2004).

De acordo com a literatura, a maneira como a conjugalidade se expressa na parentalidade pode ser entendida através do conceito denominado spillover ${ }^{4}$ (Erel \& Burman, 1995); (Krishnakumar \& Buehler, 2000). O conceito spillover é originado de distintas orientações teóricas como a teoria do estresse (Conger et al., 1992a, 1993b), a teoria da aprendizagem social (Patterson, 1989), a teoria ecológico-sistêmica (Brofrenbrenner, 1996) e a teoria dos sistemas familiares (Minuchin, 1982).

A hipótese spillover sustenta uma relação de influência direta, ou seja, a forma como se estabelecem as relações conjugais terão conseqüências que transbordarão e atingirão a relação pais e filhos. Assim, se as relações conjugais se estabelecem de forma negativa seus efeitos serão diretos e influenciarão negativamente os filhos (Erel \& Burman, 1995).

No início da década de 2000, Krishnakumar e Buehler realizaram uma meta-análise com objetivo de comprovar os achados de Erel e Burman na década de noventa. Os pesquisadores encontraram associação entre o conflito conjugal e práticas parentais ineficazes, as correlações mostraram-se mais fortes entre o conflito conjugal, práticas punitivas e falta de aceitação afetiva com os filhos (Krishnakumar \& Buehler, 2000).

\footnotetext{
${ }^{4}$ Spillover: pode ser traduzido como transbordar.
} 
Nesta perspectiva, no final dos anos noventa, Almeida, Wetherington e Chandler (1999) já haviam apontado essa relação ao identificar, através de uma pesquisa com anotações diárias sobre a vida conjugal e parental de casais norte-americanos, que tanto as mães quanto os pais mostraram-se $50 \%$ mais propensos a interagir de forma agressiva e menos responsiva com seus filhos, no dia em que haviam tido algum desentendimento com seu cônjuge.

Esses resultados sustentam o entendimento da teoria da aprendizagem social (Patterson, 1989) de que um casal com poucas habilidades em resolver problemas conjugais, definido como inabilidade em colaborar com o outro, comunicar-se de forma positiva e ser capaz de regular afetos negativos, não terá também essas habilidades como progenitores e conseqüentemente, terá pouca capacidade de responder de forma afetiva e consistente as necessidades de seus filhos (Webster-Stratton \& Hammond, 1999).

$\mathrm{O}$ estudo longitudinal desenvolvido por Gerard, Krishnakumar e Buheler (2006), investigou as relações entre o conflito conjugal, a parentalidade e o ajustamento dos filhos, através de uma amostra de 551 pais norte-americanos e mostrou que a relação entre essas variáveis se mantiveram estáveis do período da meia-infância até a adolescência de seus filhos.

Entretanto, os resultados mostram que as conexões são estáveis tanto para o conflito conjugal e a hostilidade parental, como também para baixos níveis de conflito e altos níveis de envolvimento parental. Isso indica que os filhos são atingidos tanto pelas dimensões negativas quanto positivas da conjugalidade.

Nesta perspectiva, identifica-se que, atualmente, os pesquisadores começaram a desenvolver observações mais detalhadas das interações conjugais (Cummings \& Davies, 2002); (Davies, Cummings \& Winter, 2004) precisamente, para tentar identificar os processos que conectam a relação conjugal com a parentalidade. Esses estudos mostraram a importância de avançar das perspectivas individuais e globais para a investigação dos modelos de interação entre as variáveis.

$\mathrm{O}$ esforço atual dos pesquisadores centra-se em identificar de forma mais complexa esses modelos de interação. Essas novas direções consideram a atuação de múltiplos fatores de influencia e efeitos ao longo do tempo (Gerard, Krishnakumar \& Buehler, 2006). Em suma, essa chamada "segunda geração" (Cummings \& Davies, 2002) de investigações procura ampliar o entendimento dos processos e conexões entre os dois subsistemas: conjugal e parental.

Neste sentido, diversas pesquisas norte-americanas passaram a propor modelos de relação entre determinadas dimensões da conjugalidade, da parentalidade e seus reflexos no ajustamento infantil. A maior parte dessas investigações analisa a relação entre o conflito, práticas parentais coerciti- 
vas e o ajustamento infantil (Webstter-Stratton \& Hammond, 1999); (Buehler \& Gerard, 2002). Porém, identifica-se uma lacuna nas investigações ao não considerarem outras dimensões da conjugalidade tais como a adaptabilidade, a coesão e a satisfação conjugal, variáveis que possuem relação comprovada com o funcionamento conjugal e familiar (Lindahl \& Malik, 1999); (Johnson, 2002); (Mosmann \& Wagner, 2008).

A pesquisa realizada por Johnson (2002), com adultos jovens norte-americanos, mostrou que as famílias onde se identificam relacionamentos baseados na coesão e que demonstram bons níveis de adaptabilidade, são núcleos favorecedores do desenvolvimento de filhos sensíveis a tais características. $\mathrm{O}$ autor pesquisou a associação que estes adultos jovens fazem entre as características do relacionamento conjugal de seus pais (adaptabilidade, coesão e conflito) e a sua relação com os mesmos. O pesquisador identificou que os adultos jovens que percebem e definem seus pais com capacidade de resolução de conflitos, apresentando compromisso, argumentação e negociação entre eles, também se auto-avaliam como pessoas organizadas e dotadas das mesmas características.

Neste mesmo estudo, altos níveis de adaptabilidade e coesão mostraram-se associados a baixos níveis de conflito familiar. Jovens que reportaram baixos níveis de coesão e adaptabilidade entre seus pais, foram mais propensos a relatar conflito verbal e físico entre eles e seus pais que os jovens que relataram médios e altos níveis de coesão e adaptabilidade entre seus pais. Isso sugere que se os pais são capazes de manter um relacionamento próximo afetivamente e compreensivo com seu cônjuge, os jovens tendem a perceber o ambiente familiar como coeso e afetivo, onde normalmente, se utiliza técnicas de resolução de conflito baseadas na argumentação.

Esses resultados sugerem um panorama interessante de interação entre a conjugalidade e parentalidade, assumindo a relação entre a adaptabilidade e a coesão, com o conflito conjugal, podemos pensar também que essa associação se expressa em relação à satisfação conjugal. Esses casais que possuem altos níveis de adaptabilidade e coesão, provavelmente, experimentam altos níveis de satisfação conjugal, e em conseqüência, baixos níveis de conflito. Sendo assim, todas essas dimensões em interação parecem se refletir em práticas educativas mais responsivas e menos coercitivas (Mosmann \& Wagner, 2008).

Seguindo essa linha de entendimento, Olson e DeFrain (2000) propuseram uma integração do modelo Circumplexo (Olson, Sprenkle, \& Russell, 1979); (Olson, Russell \& Sprenkle, 1983); (Olson, 2000) com os estilos educativos parentais de Baumrind (1965a, 1971b, 1978c, 1996d).

No modelo circumplexo de Olson, a coesão é definida como o grau de conexão emocional entre os membros do casal. Níveis equilibrados de 
coesão indicam um relacionamento saudável com senso de proximidade afetiva e com independência entre os cônjuges. Níveis muito alto ou muito baixos de coesão indicariam problemas para o funcionamento conjugal.

A adaptabilidade é definida como a capacidade do casal de mudar e se adaptar em resposta a problemas situacionais ou do ciclo vital familiar. Assim como a coesão, níveis equilibrados de adaptabilidade estariam associados a um melhor funcionamento conjugal e níveis extremos, muito alto ou muito baixos a dificuldades no relacionamento conjugal.

Com relação aos estilos parentais, a tipologia proposta por Baumrind (1965) classifica o tipo de aceitação afetiva (responsividade) e o tipo de controle (exigência) exercido pelos pais através de três possibilidades: autoritário, autorizante ou permissivo.

Embora esta classificação tenha sido utilizada em diversas pesquisas nos anos oitenta, Maccoby e Martin (1983) propuseram uma ampliação deste entendimento através das dimensões de responsividade e exigência e desmembraram o estilo permissivo em negligente e indulgente. Isto se baseou no entendimento destes pesquisadores de que pais que possuem baixos níveis de exigência podem variar na intensidade da responsividade. Assim, o estilo permissivo foi decomposto em negligente e indulgente.

Desta forma a classificação dos autores (Maccoby \& Martin, 1983) define que pais com altos níveis de responsividade e exigência são classificados como autorizantes; em contraponto pais que apresentam baixos níveis de responsividade e exigência são tidos como negligentes. Pais com altos índices de responsividade, mas pouco exigentes são categorizados como indulgentes, enquanto os que apresentam altos níveis de exigência e pouca responsividade são tidos como autoritários.

$\mathrm{O}$ entendimento de que as relações entre as dimensões da conjugalidade e da parentalidade necessitam de mais modelos explicativos de interação propiciou esta integração proposta por Olson e DeFrain (2000). Os autores associaram teoricamente o estilo autorizante (alta responsividade e alta exigência) a níveis equilibrados da dimensão adaptabilidade e da dimensão coesão no subsistema conjugal. $\mathrm{O}$ estilo permissivo (alta responsividade e baixo controle) foi relacionado a níveis muito altos de adaptabilidade e coesão conjugal. O estilo autoritário (alta exigência e baixa responsividade) foi associado a baixos níveis de adaptabilidade, mas índices muito altos de coesão entre os cônjuges.

Esse entendimento teórico foi testado na pesquisa realizada por Mupinga, Garrison e Pierce (2002), com 151 mães norte-americanas que teve o objetivo de examinar a relação entre o funcionamento conjugal e os estilos educativos parentais. Os resultados mostraram de forma geral, que níveis médios de coesão e adaptabilidade relatados pelas mães, se relacionaram positivamente com o estilo parental autorizante desempenhado por elas, 
e que níveis muito altos dessas dimensões associaram-se ao estilo parental autoritário. Neste estudo não houve associação significativa entre as dimensões do funcionamento conjugal e o estilo parental permissivo.

Embora os resultados não possam ser generalizados, neste estudo os níveis médios das dimensões adaptabilidade e coesão da relação conjugal destas mães apresentaram relação significativa com o estilo parental autorizante e mostraram ser um preditor mais potente que suas variáveis sócio-demográficas (idade, nível de escolaridade e renda pessoal). Ou seja, as mães que apresentaram essas características de dinâmica conjugal são de forma geral mais responsivas no relacionamento com seus filhos. Esse padrão de interação deriva de um casal que possui flexibilidade para se adaptar as circunstâncias e também, proximidade afetiva entre os membros. Esse funcionamento pode resultar em uma família com melhor capacidade para enfrentar as dificuldades do contexto em que está inserida.

De forma geral, os resultados deste estudo sustentam a integração realizada por Olson e DeFrain's (2000), entretanto, o fato de algumas associações não terem sido comprovadas estatisticamente, pode ser resultado da interação de outras variáveis familiares nesse processo, que não foram estudadas. Neste sentido, podemos pensar na variável conflito, já devidamente comprovada como fundamental no entendimento desse processo de relação (El-Sheik \& El-More Staton, 2004); (Gerard, Krishnakumar \& Buehler, 2006), assim como a satisfação conjugal (Rosen-Grandon, Myers \& Hatti, 2004).

$\mathrm{O}$ modelo de relação interativo e bidirecional entre estas dimensões da conjugalidade e da parentalidade foi proposto por Mosmann e Wagner (2008) ao comprovarem a correlação entre a adaptabilidade, a coesão, a satisfação, o conflito conjugal e as dimensões responsividade e exigência.

Este modelo sustenta-se na hipótese spillover entendida à luz da teoria ecológico - sistêmica, da teoria dos sistemas familiares (Minuchin, 1982) e da teoria da aprendizagem social (Patterson, 1989), considerando a interdependência dos subsistemas familiares e a correlação bi-direcional entre as variáveis da conjugalidade e da parentalidade (Mosmann \& Wagner, 2008).

A comprovação da existência de correlação entre as dimensões propostas no modelo nos aponta para necessidade de avançar nesse entendimento no sentido de perfilar melhor essas associações. Identifica-se a necessidade de entender qual o papel desempenhado por cada uma das dimensões da conjugalidade em relação às variáveis da parentalidade. Mais especificamente, faz-se relevante buscar analisar semelhanças e diferenças entre os casais através das dimensões da conjugalidade e sua expressão na parentalidade.

Neste sentido, considerando as dimensões de responsividade e exigência do modelo que compõem a classificação dos estilos educativos pa- 
rentais, autorizante, autoritário, indulgente e negligente, buscou-se no presente estudo traçar um perfil discriminante entre variáveis sócio-demogrãficas, a adaptabilidade, a coesão, a satisfação e o nível de conflito dos casais em relação aos estilos educativos parentais exercidos com seus os filhos.

\section{Método}

\section{Amostra}

Participaram deste estudo 149 casais, com pelo menos um filho adolescente (idade entre 13 e 19 anos) proveniente desta união, com idade média de 45,7 anos e de nível sócio-econômico médio, residentes na capital e no interior do Rio Grande do Sul/Brasil. A seleção da amostra seguiu o critério de conveniência. Em relação à escolaridade, 35,6\% dos casais possuíam nível médio, $28,9 \%$ ensino superior e $22,8 \%$ pós-graduação. A maioria dos casais $85,2 \%$ eram casados oficialmente e $14,8 \%$ viviam em união estável. O número de filhos variou de 1 a 6 sendo que a maior parte 50,7\% possuíam 2 filhos.

De forma geral, a amostra caracterizou-se por ser bastante homogênea quanto à situação conjugal, mas heterogênea em termos de escolaridade, renda pessoal e número de filhos.

\section{Instrumentos}

Os membros dos casais responderam a um instrumento composto de um questionário e quatro escalas.

Parte I - Dados de Identificação

Foram coletadas informações relativas à idade, nível de escolaridade, ocupação atual, carga horária de trabalho e renda pessoal. Ademais, investigou-se dados sobre o companheiro/a e a família do sujeito.

Parte II - Escala de Satisfação Conjugal - The Golombok Rust Inventory of Marital State - GRIMS

O GRIMS (Rust et al., 1988, traduzida e adaptada a língua portuguesa por Falcke, 2003) é constituído por 28 itens, os quais o sujeito deve pontuar em uma escala Likert de 4 pontos (discordo fortemente, discordo, concordo e concordo fortemente). Esta escala mede a qualidade do relacionamento conjugal através de dimensões que são consideradas importantes para um bom relacionamento. São elas: satisfação, comunicação, interesses compartilhados, confiança e respeito. Na pontuação da escala, verifica-se que quanto maiores os escores obtidos, mais severos são os problemas no relacionamento conjugal. O coeficiente Alpha de Cronbach obtido para o GRIMS foi de 0,80 . 
Parte III - Escala de avaliação da coesão e adaptabilidade familiar Faces III.

O Faces III é uma escala com vinte itens pontuados em uma escala Likert de 5 pontos (quase nunca, alguma vez, às vezes, com freqüência, quase sempre) para avaliar a coesão e adaptabilidade familiar e conjugal (Olson, 1979, traduzido e adaptado por Falceto, 1997).

O procedimento de pontuação realiza-se, na dimensão de coesão, através da soma dos itens ímpares e, na dimensão adaptabilidade, pela soma de todos os itens pares (Córdoba, 1989).

O coeficiente Alpha de Cronbach obtido para a dimensão coesão foi de 0,78 e para adaptabilidade 0,72 .

Parte IV - Escala de Conflito Conjugal

A escala é constituída por 9 itens que são apresentados separadamente devido ao enunciado ficando assim, dividida em duas sub-escalas. A primeira denominada "conflito-desentendimentos" possui 6 itens que se referem à freqüência com que os sujeitos experimentaram desentendimentos com seus parceiros no ultimo ano medida em uma escala Likert de 6 pontos (nunca, uma vez ao mês ou menos, diversas vezes ao mês, aproximadamente uma vez por semana, diversas vezes por semana, quase todos os dias). A outra sub-escala denominada "conflito-agressão" possui 3 itens que são pontuados em uma escala Likert de 5 pontos (nunca, raramente, algumas vezes, freqüentemente, sempre) 1 item mede a freqüência com que o sujeito lida de forma calma com os conflitos (codificado invertido) e dois itens medem a freqüência de discussões e agressões. Possui um escore médio e os escores maiores representam altos níveis de conflito (Buehler \& Gerard, 2002).

O coeficiente Alpha de Cronbach obtido para a escala "conflito-agressão" de foi de 0,71 e para escala "conflito-desentendimentos" foi de 0,77 .

Parte V - Escala de Estilos Parentais

A escala de estilos parentais foi desenvolvida por Lamborn et al. (1991) com objetivo de classificar as práticas parentais dentro das dimensões de responsividade e exigência propostas por Maccoby \& Martin (1984).

No presente estudo foi utilizada a última versão traduzida e adaptada ao português por Teixeira et al. (2004). Os índices de consistência interna encontrados foram muito bons sendo o alpha de cronbach da escala de responsividade 0,82 e da escala de exigência 0,73 .

Através destas duas sub-escalas pontuadas em uma escala Likert de 4 pontos (quase nunca, raramente, às vezes, geralmente, quase sempre) se obtém uma classificação das práticas parentais dentre os quatro estilos teo- 
ricamente definidos: autoritário, autorizante, negligente e indulgente (Lamborn et al., 1991).

\section{Procedimentos Para Coleta de Dados}

Realizou-se uma seleção de voluntários para participarem da coleta desta pesquisa na Pontifícia Universidade Católica do Rio Grande do Sul (PUCRS). Uma vez escolhidos os voluntários, estes foram treinados para aplicar os questionários nos participantes. Os voluntários eram provenientes da cidade de Porto Alegre/RS e do interior do Estado do Rio Grande do $\mathrm{Sul} / \mathrm{RS}$, o que fez com que a coleta fosse realizada em diversas cidades do estado.

O voluntário fazia contato com os casais explicando sobre o tema da pesquisa e se houvesse interesse do casal em participar, marcava um encontro para aplicação do instrumento.

Neste encontro o voluntário entregava um envelope para cada cônjuge onde estavam os questionários, juntamente com uma carta com instruções sobre a pesquisa e o termo de consentimento livre e esclarecido.

O casal era orientado a responder separadamente os questionários e após terminarem, devolviam ao aplicador junto ao termo de consentimento livre e esclarecido assinado, que os colocava no envelope e o lacrava diante do casal para garantir o sigilo dos participantes.

\section{Considerações Éticas}

O presente estudo foi aprovado pelo Comitê de Ética e Pesquisa da Pontifícia Universidade Católica e seguiu todos os procedimentos éticos recomendados.

\section{Apresentação e Discussão dos Resultados}

Foi realizada uma análise discriminante que buscou determinar de que forma as variáveis sócio-demográficas e da conjugalidade, diferenciam os participantes do estudo em relação aos quatro estilos educativos parentais - autorizante, autoritário, indulgente e negligente -, no intuito de obter um perfil discriminante dos casais investigados.

A análise discriminante utiliza uma variável de agrupação que maximiza a distância (diferença) entre os grupos, no caso, os estilos educativos parentais. O número de funções discriminantes é igual ao número de grupos menos um, no entanto nem todas têm o mesmo poder explicativo (Sarriera, 1996). Por esse motivo, serão apresentadas as três funções obtidas, mas 
apenas as duas com maior poder explicativo serão discutidas detalhadamente.

Foram incluídas 13 variáveis preditoras na análise dos quatro estilos educativos parentais: adaptabilidade, coesão, satisfação conjugal, conflito-desentendimentos, conflito-agressão, sexo, escolaridade, carga horária de trabalho, renda pessoal, horas que fica com filho durante a semana, horas que fica com filho durante o final de semana, horas que fica com marido durante a semana e horas que fica com marido durante o final de semana.

Foram incluídas essas variáveis de acordo com o critério de que, na análise discriminante, apenas insere-se variáveis quantitativas. Essas variáveis geraram três funções discriminantes apresentadas na Tabela 1:

Tabela 1: Auto-Valor das funções obtidas.

\begin{tabular}{lccccccccc}
\hline Função & $\begin{array}{c}\text { Auto- } \\
\text { valor }\end{array}$ & $\begin{array}{c}\text { \% da } \\
\text { variân- } \\
\text { cia }\end{array}$ & $\begin{array}{c}\text { Variân- } \\
\text { cia } \\
\text { Cumu- } \\
\text { lativa }\end{array}$ & $\begin{array}{c}\text { Corre- } \\
\text { lação } \\
\text { Canô- } \\
\text { nica }\end{array}$ & & & & & \\
& & & & & & & & \\
\hline $\mathbf{1}$ & 0,273 & $56,7 \%$ & $56,7 \%$ & 0,463 & 0,646 & 125,984 & 39 & 0,000 \\
$\mathbf{2}$ & 0,259 & $33,0 \%$ & $89,7 \%$ & 0,370 & 0,822 & 56,443 & 24 & 0,000 \\
$\mathbf{3}$ & 0,049 & $10,3 \%$ & $100 \%$ & 0,217 & 0,953 & 13,895 & 11 & 0,239 \\
\hline
\end{tabular}

A função 1 apresentou maior poder explicativo da variância $(56,7 \%)$ em relação a função 2 que mostrou $33,0 \%$ de força explicativa. A função 3 indicou o menor poder explicativo (10,3\%) e não apresentou diferença estatística significativa $(p=0,239)$. Por esse motivo, iremos analisar mais detalhadamente os resultados das funções 1 e 2.

Para compreender melhor o que cada uma das funções está discriminando utilizaram-se os valores dos centróides em cada uma das funções. Os valores dos centróides da função 1 foram 0,283 para o estilo autorizante, $-0,494$ para o estilo negligente, $-0,621$ para o estilo autoritário e 0,709 para o estilo indulgente. Na função 2 os valores dos centróides foram 0,386 para o estilo autorizante, $-0,465$ para o estilo negligente, 0,324 para o estilo autoritário e -0,382 para o estilo indulgente.

A função 1 foi denominada Responsividade por diferenciar, com maior poder explicativo, entre os estilos alto em responsividade (autorizante e indulgente) e os estilos baixos em responsividade (negligente e autoritário). A função 2 foi chamada Exigência por discriminar entre os estilos bai- 
xos em exigência (indulgente e negligente) e os estilos altos em exigência (autoritário e autorizante).

A função 1, por apresentar um maior poder explicativo $(56,7 \%$ da variância), será analisada primeiramente. A seguir apresenta-se a matriz estrutural que indicou que variáveis são as mais relevantes na capacidade discriminatória da função (Tabela 2).

Tabela 2: Matriz Estrutural da função Responsividade.

\begin{tabular}{lc}
\hline Variáveis & Função Responsividade \\
\hline Coesão & 0,592 \\
Satisfação Conjugal & 0,502 \\
Adaptabilidade & 0,490 \\
Sexo do Cônjuge & $-0,428$ \\
Carga horária de trabalho & $-0,328$ \\
Conflito-agressão & $-0,306$ \\
Escolaridade & 0,266 \\
Conflito-desentendimentos & $-0,241$ \\
Horas que passa com cônjuge durante o & 0,241 \\
final de semana & \\
Horas que passa com o cônjuge durante a & 0,077 \\
semana & \\
Renda pessoal & 0,049 \\
Horas que passa com filho durante a & $-0,043$ \\
semana & \\
Horas que passa com filho durante o & 0,023 \\
final de semana & \\
\hline
\end{tabular}

\section{Função Responsividade}

Os valores da tabela 5 mostram as variáveis agrupadas que apresentaram maiores níveis de correlação com a função discriminante Responsividade. 
Identifica-se que, para os casais dessa amostra, em relação à função responsividade, a coesão (,592), a adaptabilidade (,490), assim como a satisfação conjugal $(, 502)$, apresentam robusta capacidade discriminatória, em maior grau do estilo indulgente e também do autorizante, em relação aos estilos baixos em responsividade, autoritário e negligente.

Especificamente, quanto mais altos os níveis de coesão e adaptabilidade conjugal maior a diferença entre o estilo educativo parental indulgente e o autoritário. Pode-se considerar que são casais com níveis muito altos de proximidade afetiva e muito flexíveis, o que se reflete em um estilo parental com alta responsividade, mas baixo nível de exigência com os filhos. Esse dado está em consonância com o modelo proposto por de Olson e DeFrain's (2002) de integração das dimensões de adaptabilidade e coesão aos estilos educativos parentais. Nesse entendimento o estilo permissivo (alto em responsividade e baixo em exigência) aparece associado a níveis muito altos de coesão e adaptabilidade.

Ademais, esses casais também apresentam altos níveis de satisfação conjugal, o que, provavelmente, é resultado de seu alto nível de proximidade afetiva e grande capacidade de adaptação, o que possivelmente faz com que consigam lidar com os desafios da vida conjugal. Entretanto, parece que esses altos níveis de adaptabilidade se refletem em uma dificuldade de monitorar o comportamento dos filhos, sendo muito responsivos com eles, mas com pouco controle sobre seus comportamentos.

O estilo educativo indulgente por apresentar alta responsividade, aparece em pesquisas nacionais (Teixeira, Bardagi, Gomes, \& Hutz, 2004); (Predrebon, 2005) e internacionais (Aunola, Sttatin \& Nurmi, 2000); (Slicker, Picklesimer, Guzak, Fuller, 2005), associado a índices de bem-estar psicológico, auto-estima, autoconfiança e desenvolvimento de habilidades interpessoais dos filhos. A ênfase aqui parece ser a responsividade, pois mesmo os pais tendo baixos níveis de exigência com seus filhos, estes terminam por apresentar bons níveis de ajustamento. Observa-se que a aceitação afetiva por parte dos pais faz a diferença nesse processo, sendo assim, essa proximidade afetiva parece iniciar-se com o cônjuge e ser extendida ao relacionamento com os filhos.

Nesta perspectiva, identifica-se que níveis não tão altos de coesão e adaptabilidade conjugal, associados a uma relação positiva com a função responsividade, demonstram discriminar mais o estilo parental autorizante, em relação aos demais. Ou seja, casais com níveis equilibrados de proximidade afetiva e capacidade de adaptação além de serem responsivos com seus filhos, também são capazes de monitorar seus comportamentos com eficácia (estilo autorizante). Em relação à função Responsividade, identifica-se que o equilíbrio das dimensões da conjugalidade, adaptabilidade e coesão, diferenciam também o estilo parental que equilibra a responsividade 
e a exigência. No entendimento de Patterson (1989) esses seriam casais que possuem habilidades pessoais às quais fazem com que sejam capazes de regular seus afetos e resolver problemas tanto com seu cônjuge quanto com seus filhos.

O fato das correlações entre as variáveis e a função discriminante serem positivas ou negativas não é mais relevante do que o poder explicativo das mesmas. Dessa forma, as variáveis que apresentaram médio poder explicativo (acima de 0,30 ) da função responsividade foram o sexo do cônjuge $(-0,428)$, a carga horária de trabalho $(-0,328)$ e conflito-agressão $(-0,306)$.

A variável sexo tem um valor negativo nessa função, e pelo fato da classificação entre os sexos utilizada neste estudo definir o sexo feminino com o valor 1 e o masculino com o valor 2, pode-se dizer que as mulheres/mães discriminam de forma mais consistente o estilo negligente em relação ao indulgente. Ou seja, entre os estilos baixos em responsividade dos estilos altos em responsividade.

Podemos traçar um perfil discriminante em relação às variáveis que apresentaram médio poder explicativo da função responsividade. Identificámos que o sexo do cônjuge, a carga horária de trabalho e o conflito agressão são as variáveis que discriminam esses casais em relação aos estilos educativos baixos em responsividade. Nesse caso, as mulheres que trabalham poucas horas semanais, têm baixos níveis de conflito com seus cônjuges são as que, neste estudo, adotam o estilo educativo negligente. Aqui novamente surge uma diferença em termos de exigência. Parece que as mulheres desta amostra, apesar de disporem de tempo para se ocuparem da educação dos filhos, não se mostram nem responsivas e nem exigentes para com eles. Provavelmente, essa indiferença expressa-se na vida do casal de forma geral, elas talvez estejam pouco envolvidas em seus trabalhos, com o cônjuge e, da mesma forma, os filhos fiquem relegados a um segundo plano (Webster-Stratton \& Hammond, 1999).

Outras variáveis mostraram-se associadas à função Responsividade, mas em menor grau de correlação. São elas: o nível de escolaridade, conflito - desentendimentos, o tempo que passam com o cônjuge no final de semana e durante a semana, a renda pessoal, e o tempo que passam com os filhos durante a semana e no final de semana.

Em resumo, podemos traçar um perfil discriminante dos casais a partir das variáveis que apresentaram maior poder explicativo da função responsividade. Identifica-se que casais com altos níveis de coesão, adaptabilidade e satisfação conjugal tendem a exercer um estilo educativo alto em responsividade.

Podemos identificar que níveis extremos dessas variáveis tendem a discriminar mais o estilo indulgente caracterizado por muita responsividade, 
e pouco controle. Neste caso, são casais muito próximos afetivamente, entretanto não monitoraram o comportamento dos filhos. Talvez, sejam casais que enfoquem mais o afeto tanto com o cônjuge quanto com os filhos, em detrimento da exigência e do controle.

\section{Função Exigência}

A função Exigência também apresentou um bom poder explicativo, $33 \%$. A seguir apresentamos a matriz estrutural desta função (Tabela 3).

Tabela 3: Matriz Estrutural da função Exigência

\begin{tabular}{lc}
\hline Variáveis & Função Exigência \\
\hline Renda Pessoal & $-0,661$ \\
Horas que passa com filho durante o final de semana & 0,434 \\
Horas que passa com filho durante a semana & 0,400 \\
Conflito-desentendimentos & 0,297 \\
Adaptabilidade & 0,296 \\
Coesão & 0,269 \\
Escolaridade & $-0,225$ \\
Carga horária de trabalho & $-0,159$ \\
Satisfação Conjugal & 0,146 \\
Conflito-agressão & $-0,126$ \\
Sexo do Cônjuge & $-0,103$ \\
Horas que passa com marido final de semana & 0,056 \\
Horas com marido durante a semana & 0,024 \\
\hline
\end{tabular}

Podemos considerar que esse perfil talvez nos forneça um panorama dos pais que, na atualidade, apresentam tantas dificuldades em dar limites aos filhos. Pode-se perfilar então um grupo de casais que estão satisfeitos em seus relacionamentos conjugais, têm muito afeto por seus filhos, mas são muito flexíveis, e possuem pouco controle sobre eles. Talvez, esses pais não tenham claro que, monitoramento e controle brandos são também demonstrações de interesse e cuidado para com os filhos.

Quando pensamos em níveis moderados dessas variáveis elas parecem diferenciar o estilo autorizante dos demais. Identifica-se que aqui surge 
um equilíbrio entre a responsividade e a exigência. Este é o grupo de casais que consegue balancear a quantidade de afeto e de flexibilidade com bons níveis de satisfação conjugal. Esse equilíbrio parece se expressar na forma como o casal educa seus filhos e pode se refletir em uma família com melhor capacidade para enfrentar as circunstâncias tanto no micro, quanto no macrosistema familiar.

Todos esses perfis corroboram a hipótese spillover de relação direta e dinâmica entre a conjugalidade e a parentalidade. As variáveis que utilizamos neste estudo para definir a qualidade conjugal (adaptabilidade, coesão, satisfação conjugal e conflito) se refletem de forma expressiva nos estilos educativos que adotam os casais dessa amostra.

Assim, a coerência demonstrada neste estudo entre a forma de funcionamento do subsistema conjugal com o parental remete-nos às características individuais dos sujeitos que compõe tais subsistemas. Certamente, suas habilidades pessoais se expressam positivamente nas funções que exercem na família. Neste caso, quando cônjuges, o afeto e a flexibilidade se traduzem pela coesão e a adaptabilidade e quando, pais se expressam através da responsividade e da exigência para com os filhos.

Os valores da Tabela 4 mostram as variáveis que, reunidas, apresentaram maiores níveis de correlação com a função discriminante Exigência.

As variáveis que apresentaram maior poder explicativo da função foram a renda pessoal $(-0,661)$, horas que passa com o filho durante a semana $(0,434)$, horas que passa com o filho durante o final de semana $(0,400)$.

A renda pessoal discriminou mais fortemente o estilo educativo negligente em relação ao autoritário. Especificamente, em relação ao estilo autoritário, que diz respeito à baixa responsividade e alta exigência parental, parece que os casais dessa amostra, com menor poder econômico tendem a uma maior utilização de um estilo mais rígido no processo de socialização de seus filhos. Esses resultados são confirmados pela literatura uma vez que, de acordo com pesquisas, pais com menores ingressos econômicos tendem a utilizar mais estratégias punitivas e coercitivas com seus filhos (Ceballos \& Rodrigo, 1998); (Mupinga, Garrison \& Pierce, 2002), o que se relaciona mais ao estilo autoritário.

Em relação às outras duas variáveis, identifica-se que o perfil discriminante desses casais indica que o tempo que passam junto com os seus filhos é o que mais diferencia entre o estilo educativo autoritário (alto em exigência) e o estilo negligente (baixo em exigência). Nessa situação, observa-se que o tempo dedicado aos filhos aumenta a demanda parental, tornando a relação ainda mais complexa, devido à exigência de um maior repertório de respostas às demandas dos filhos. Neste sentido, estratégias autoritárias e pouco flexíveis podem estar representando a necessidade de 
um controle imediato da situação. Entretanto, está comprovado que um controle excessivo por parte dos pais pode levar a uma vida familiar muito rígida (Slicker, Picklesimer, Guzak, Fuller, 2005).

Ademais, desde o entendimento da hipótese spillover e do modelo de correlação bi-direcional entre as variáveis da conjugalidade e da parentalidade (Mosmann \& Wagner, 2008) podemos pensar que casais que tendem a utilizar práticas educativas mais punitivas, normalmente são pessoas com dificuldade em resolver problemas e regular afetos tanto com seu cônjuge quanto com seus filhos (Patterson, 1989; Gerard, Krishnakumar \& Buehler, 2006).

Em relação às demais variáveis com menor poder discriminante, identificamos um perfil diferente de casais. Nota-se que o que mais discrimina o estilo autorizante dos demais, em relação à exigência, é o conflito conjugal acerca de desentendimentos, a adaptabilidade e a coesão. Talvez aqui, possamos pensar em casais com capacidade de flexibilidade, suficientemente próximos emocionalmente, que lidam com os conflitos de forma adaptativa e não de maneira disfuncional, e que são ao mesmo tempo, responsivos e exigentes com seus filhos.

As demais variáveis apresentam um baixo poder discriminante em relação à função exigência. São elas: escolaridade, carga horária de trabalho, satisfação conjugal, conflito - agressão, sexo e as horas que passa com o marido durante a semana e no final de semana.

De forma geral, podemos identificar que os perfis discriminantes dos casais em relação à função exigência diferenciam-se basicamente pela renda pessoal. Os casais com baixos ingressos pessoais tendem a utilizar mais estratégias punitivas e coercitivas com seus filhos, além de estarem bastante tempo em sua companhia tanto nos dias de semana, quanto nos finais de semana. Por outro lado, os casais com maiores ingressos pessoais, bons níveis flexibilidade e proximidade afetiva, embora com alguns desentendimentos, são aqueles que apresentam o estilo parental autorizante, ou seja, são pais que acreditam que devem estimular os filhos a desenvolver autonomia, mas não são permissivos em relação à desobediência das normas que estabelecem.

Nesta perspectiva, observa-se que, para os casais desta amostra, maior renda pessoal associa-se a maior capacidade dos sujeitos vivenciarem uma conjugalidade com maior capacidade adaptativa e proximidade afetiva. Sendo assim, estes aspectos terão expressão na parentalidade através de bons níveis de responsividade e exigência.

Além das variáveis correlacionadas às funções discriminantes, estatisticamente é bastante relevante saber qual é a capacidade final da função discriminante para classificar corretamente aos sujeitos no seu grupo correspondente (Sarriera, 1996). Isso fica evidenciado na tabela abaixo: 
Tabela 4: Classificação dos estilos educativos parentais

\begin{tabular}{|c|c|c|c|c|c|c|c|}
\hline & & \multicolumn{6}{|c|}{$\begin{array}{l}\text { Grupo prognosticado de per- } \\
\text { tencimento }\end{array}$} \\
\hline & & Estilos & $\begin{array}{l}\text { Auto- } \\
\text { rizante }\end{array}$ & $\begin{array}{l}\text { Negli- } \\
\text { gente }\end{array}$ & $\begin{array}{l}\text { Auto- } \\
\text { ritário }\end{array}$ & $\begin{array}{l}\text { Indul- } \\
\text { gente }\end{array}$ & Total \\
\hline \multirow[t]{8}{*}{ Original } & Recontagem & Autorizante & 55 & 12 & 14 & 22 & 103 \\
\hline & & Negligente & 11 & 32 & 18 & 14 & 75 \\
\hline & & Autoritário & 10 & 12 & 30 & 6 & 58 \\
\hline & & Indulgente & 10 & 8 & 7 & 37 & 62 \\
\hline & & Autorizante & 53,4 & 11,7 & 13,6 & 21,4 & 100 \\
\hline & $\%$ & Negligente & 14,7 & 42,7 & 24,0 & 18,7 & 100 \\
\hline & & Autoritário & 17,2 & 20,7 & 51,7 & 10,3 & 100 \\
\hline & & Indulgente & 16,1 & 12,9 & 11,3 & 59,7 & 100 \\
\hline
\end{tabular}

Nota: * Classificados corretamente $51,7 \%$ dos casos agrupados originalmente.

A tabela expressa um procedimento de validação da função, já que resume a capacidade preditiva da função discriminante (Pardo y Ruiz, 2002). O estilo indulgente foi classificado corretamente em $59,7 \%$ dos casos, o autorizante em 53,4\% dos casos, o autoritário em 51,7\% e o negligente em $42,7 \%$ dos casos. No total, a função consegue classificar corretamente $51,7 \%$ dos casos estudados. Esse dado deve ser interpretado com base na classificação correta esperada ao acaso. Dessa forma, como são quatro estilos educativos parentais, a expectativa de classificação correta ao acaso seria de $25 \%$ assim, o valor de $51,7 \%$ é bastante significativo. Podemos considerar então, que para um pouco mais da metade dos casais dessa amostra, esses perfis discriminantes estão corretamente classificados.

Neste sentido, ao observarmos os perfis definidos pelas correlações entre as variáveis da conjugalidade em relação às funções discriminantes, percebe-se o padrão interativo e dinâmico entre os dois subsistemas. As dimensões que compõem e caracterizam a conjugalidade destes casais estudados se expressam de forma coerente na parentalidade.

Em realidade, evidencia-se novamente a impossibilidade de considerar isoladamente as habilidades individuais dos membros do casal para a conjugalidade sem entender a reverberação desse aspecto na dinâmica conjugal e parental. Concomitantemente, o entendimento de senso comum, que 
as dificuldades no relacionamento conjugal podem ser compensadas na dedicação à educação dos filhos parece não ser verdadeiro para os casais dessa amostra. A compreensão ecológico-sistêmica das relações familiares nos indica que as características tanto positivas quanto negativas que os cônjuges possuem como indivíduos, se expressam nas interações conjugais e parentais no mesmo sentido.

\section{Considerações Finais}

Ao analisarmos os resultados do presente estudo, identificamos que o perfil discriminante observado nestes participantes, no que diz respeito à relação existente entre a conjugalidade e os estilos educativos parentais, nos faz pensar sobre a necessidade de fazerem-se intervenções psicossociais com casais que visem à prevenção e otimização das interações familiares.

Nesta perspectiva, percebemos que a idéia do senso comum de que é melhor manter-se casado, ainda que insatisfeito, pelo "bem das crianças", não se sustenta frente à comprovação da relação existente entre a conjugalidade e a parentalidade. O pensamento de que é possível manter as crianças afastadas das dificuldades conjugais não é efetivo, uma vez que nossos achados indicam que as características da relação conjugal terão expressão nos estilos educativos desempenhados pelo casal, no exercício da parentalidade.

Ademais, o fato de esta interrelação ser efetiva, evidencia que tanto os aspectos positivos, quanto negativos da conjugalidade têm expressão na parentalidade e esse processo aponta o caminho para as intervenções familiares.

A busca por auxílio de pais com dificuldades em lidar com seus filhos, muitas vezes centra-se apenas nos sintomas dos filhos e/ou em suas habilidades como pais, o que de acordo com nossos achados, não é eficaz. As intervenções deveriam ter como objetivo desenvolver no casal efetivas habilidades de resolução de conflitos, de comunicação e formas de regular seus afetos negativos, assim como suas habilidades parentais. Ou seja, é fundamental o entendimento, de que a complexidade das relações familiares não pode ser reduzida a apenas um subsistema familiar.

Neste sentido, a relevância destes resultados está em propiciar o entendimento por parte dos cônjuges/pais, assim como dos profissionais de saúde, de que a busca por soluções práticas acerca da parentalidade, que excluem a análise de como estão se estabelecendo as relações no subsistema conjugal, tende a ser infrutífera.

Frente a esses resultados, o poder de influência dos aspectos positivos da conjugalidade em relação aos estilos educativos deveria ser enfocado de 
forma mais consistente, em detrimento dos aspectos negativos, uma vez que podem funcionar como fatores de proteção frente às dificuldades encontradas em todas as relações interpessoais. Os filhos podem aprender muito sobre como resolver seus problemas dentro e fora da família ao presenciarem a forma de resolução de conflito utilizada por seus pais, o afeto existente entre eles, como se comunicam, e principalmente como se adaptam frente às dificuldades inerentes à vida.

Algumas considerações acerca das limitações deste estudo nos apontam a necessidade de uma maior disponibilidade, em nosso contexto, de um arsenal metodológico de pesquisa que possa abranger a complexidade das interações familiares. O presente estudo, por ser de tipo transversal, e ter enfocado apenas o subsistema conjugal, provavelmente não abarcou de forma completa todas as possíveis interconexões existentes na presente amostra. Ademais, optamos por estudar casais com filhos adolescentes, e neste sentido, futuros estudos que realizassem a ampliação desta faixa etária, assim como a análise da expressão dessas relações de forma qualitativa, poderiam nos permitir um aprofundamento no entendimento desses processos.

\section{Referências}

Almeida, D. M., Wethington, E., \& Chandler, A. L. (1999) Daily transmission of tensions between marital dyads and parent-child dyads. Journal of Marriage \& theFamily, 61, 49-61.

Aunola, K., Sttatin, H., \& Nurmi, J. (2000). Parenting styles and adolescents' achievement strategies. Journal of Adolescence, 23, 205-222.

Baumrind, D. (1965). Parental control and parental love. Children, 230-234.

Baumrind, D. (1966). Effects of authoritative parental control on child behavior. Child Development, 37, 887-907.

Baumrind, D. (1971). Harmonious parents and their preschool children. Developmental Psychology, 4(1), 99-102.

Baumrind, D. (1978). Reciprocal rights and responsabilities in parent-child relations. Journal of Social Issues, 34(2), 179-197.

Bronfenbrenner, U. (1996). A ecologia do desenvolvimento humano: experimentos naturais e planejados. Porto Alegre: Artes Médicas.

Buehler, C., \& Gerard, J. M. (2002). Marital Conflict, ineffective parenting, and children's and adolescents' maladjustment. Journal of Marriage and Family, 64 (1), 78-93.

Ceballos, E., \& Rodrigo, M. J. (1998). Las metas y estrategias de socialización entre padres e hijos. In Rodrigo, M. J. \& Palacios, J. Familia y desarrollo humano. (pp. 225-243). Madrid: Alianza Editorial. 
Conger, R. D., Conger, K. J., Elder, G. H., Lorenz, F. O., Simons, R. L., \& Whitbeck, L. B. (1992). A family process model of economic hardship and adjustment of early adolescent sons. Child Development, 63, 526-541.

Conger, R. D., Conger, K. J., Elder, G. H., Lorenz, F. O., Simons, R. L., \& Whitbeck, L. B. (1993). Family economic stress and adjustment of early adolescent daughters. Developmental Psychology, 29(2), 206-219.

Córdoba, A. H. (1989). Familias Clínicas en Bogotá: su funcionamiento según el modelo circumplejo de D.H. Olson. Monografia. Bogotá.

Craddock, A. F. (1991). Relationships between attitudinal similarity, couple estructure, and couple satisfaction in married and de facto couples. Australian Journal of Psychology, 43, 11-16.

Cummings, E. M., \& Davies, P. T. (2002). Effects of marital discord on children: Recent advances and emerging themes in process-oriented research. Journal of Child Psychology and Psychiatry, 43, 31-63.

Davies, P. T., Sturge-Apple, M. L., Cummings, E. M. (2004). Interdependencies among interparental discord and parenting practices: The role of adult vulnerability and relationship perturbations. Development and Psychopathology 16, 773-797.

Davies, P. T., Cummings, E. M., \& Winter, M. A. (2004). Pathways between profiles of family functioning, child security in the interparental subsystem, and child psychological problems. Development and Psychopathology, 16, 525-550 .

El-Sheikh, M., \& Elmore-Staton, L. (2004). The marital conflict-child adjustment link: Parent-child conflict, perceived attachments, and parental depression as potentiators and mitigators of risk. Development and Psychopathology, 16, 631-648.

Erel, O., \& Burman, B. (1995). Interrelatedness of marital relations and parent-child relations: a meta-analytic review. Psychological Bulletin 118, (1), 108-132.

Escala de Conflito Conjugal. Disponível em http://ssc.wisc.edu/nsfh. Acesso em agosto de 2004.

Falceto, O. G. (1997). Famílias com desenvolvimento funcional e disfunciona: validação das escalas diagnósticas Faces III, Beavers - Timberlawn e Avaliação Global do Funcionamento Interacional (Garf). Dissertação de Mestrado. Medicina. UFRGS

Falcke, D. (2003). Águas passadas não movem moinhos?: as experiências na família de origem como preditoras da qualidade do relacionamento conjugal. Tese de Doutorado. Psicologia. PUCRS. 185f.

Frosch, C. A., \& Mangelsdorf, S. C. (2001). Marital behavior, parenting behavior, and multiple reports of preschoolers' behavior problems: Mediation or moderation? Developmental Psychology, 37(4), 502-519.

Gerard, J. M., Krishnakumar, A., \& Buheler, C. (2006). Marital Conflict, Parent-Child Relations, and Youth Maladjustment A Longitudinal Investigation of Spillover Effects. Journal of Family Issues, 27(7), 951-975. 
Jonhson, H. D. (2002). Associations among Family Adaptability and Cohesion, Interparental Conflict, and Tatics used during young adult's conflict with parents. Psychological Reports, 91, 315-325.

Krishnakumar, A., \& Buehler, C. (2000). Interparental conflict and parenting behaviors: A metaanalytic review. Family Relations, 49, 25-44.

Lamborn, S. D., Mounts, N. S., Steinberg, L., Dornbusch, S.M. (1991). Patterns of competence and adjustmentamong adolescents from authoritative, authoritarian, indulgent, and neglectful families. Child Development, 62, 1049-1065.

Lindhal, K. M., \& Malik, N. M. (1999). Marital conflict, family processes, and boy's externaling behavior in hispanic american and european families. Journal of Clinical and Child Psychology, 28(1), 12-24.

Maccoby, E., \& Martin, J. (1983). Socialization in the context of the family: Parent-child interaction. In E. M. Hetherington (Org.), P. H. Mussen (Org. Série), Handbook of child psychology: Vol. 4. Socialization, personality, and social development (4a ed., pp. 1-101). New York: Wiley.

Minuchin, S. (1982). Famílias: funcionamento e tratamento. Porto Alegre: Artes Médicas.

Mosmann, C., \& Wagner, A. Dimensiones de la Conyugalidad y de la Parentalidad: um modelo correlacional. Revista Intercontinental de Psicología y Educación, v. 10, pp. 79-103, 2008.

Mupinga, E. E., Garrison, M.E.B., Pierce, S.H. (2002). An Exploratory Study of the Relationships between Family Functioning and Parenting Styles: The Perceptions of Mothers of Young Grade School Children. Family and Consumer Sciences Research Journal, 31 (1), 112-129.

Olson, D. (2000) Circumplex Model of Marital and Family Systems. Journal of Family Therapy, 22, 144-167.

Olson, D. H., \& DeFrain, J. (2000). Marriage and the family: Diversity and strengths. Mountain View, CA: Mayfield.

Olson, D., Russell, C., \& Sprenkle, D. (1983). Circumplex model of marital and family systems: VI. Theoretical update. Family Process, 22, 69-83.

Olson, D., Sprenkle, D., \& Russell, C. (1979). Circumplex model of marital and family systems: I. Cohesion and adaptability dimensions, family types, and clinical applications. Family Process, 18, 3-15.

Pardo, A., \& Ruiz, M. A. (2002). Spss 11: Guia para el análisis de datos. Madrid: McGrawHill.

Patterson, G. R., Debaryshe, B. D., \& Ramsey, E., (1989). A developmental perspective on antisocial behavior. American Psychologist, 44 (2), 329-335.

Predebon, J. C. (2005). Variáveis preditoras dos problemas de comportamento na adolescência. Tese de Doutorado. Psicologia. PUCRS. Porto Alegre, $216 \mathrm{f}$.

Rosen-Grandon, J. R., Myers, J. E., Hatti, J. A. (2004). The Relationship Between Marital Characteristics, Marital Interaction Process and Marital Satisfaction. Journal of Counseling and Development, 82, 1, 58-68.

Rust, J., Bennun, I., Crowe, M., \& Golombok, S. (1988). The Golombok Rust Inventory of Marital State. Windsor: NFER-NELSON. 
Sarriera, J. C. (1996). Introdução à análise multivariada em psicologia. Manuscrito utilizado no curso de Pós-Graduação em Psicologia. Universidade Federal do Espírito Santo.

Slicker, E. K., Picklesimer, B. K., Guzak, A. K., Fuller, D. K. (2005). The relationship of parenting style to older adolescent life-skills development in the United States. Young, 13, 227-245.

Teixeira, M. A. P., Bardagi, M. P., Gomes, W. B., \& Hutz, C. S. (2004). Refinamento de um instrumento para avaliar responsividade e exigência parental percebidas na adolescência. Avaliação Psicológica, 3(1), 1-11.

Webster-Stratton, C., \& Hammond, M. (1999). Marital conflict management skills, parenting style, and early - onset conduct problems: processes and pathways. Journal of Child Psychology and Psychiatry, 40 (6), 917-927. 